\title{
CORRIGENDUM
}

doi:10.1038/nature24026

\section{Corrigendum: Whole-genome landscape of pancreatic neuroendocrine tumours}

Aldo Scarpa, David K. Chang, Katia Nones, Vincenzo Corbo, Ann-Marie Patch, Peter Bailey, Rita T. Lawlor, Amber L. Johns, David K. Miller, Andrea Mafficini, Borislav Rusev, Maria Scardoni, Davide Antonello, Stefano Barbi, Katarzyna O. Sikora, Sara Cingarlini, Caterina Vicentini, Skye McKay, Michael C. J. Quinn, Timothy J. C. Bruxner, Angelika N. Christ, Ivon Harliwong, Senel Idrisoglu, Suzanne McLean, Craig Nourse, Ehsan Nourbakhsh, Peter J. Wilson, Matthew J. Anderson, J. Lynn Fink, Felicity Newell, Nick Waddell, Oliver Holmes, Stephen H. Kazakoff, Conrad Leonard, Scott Wood, Qinying Xu, Shivashankar Hiriyur Nagaraj, Eliana Amato, Irene Dalai, Samantha Bersani, Ivana Cataldo, Angelo P. Dei Tos, Paola Capelli, Maria Vittoria Davì, Luca Landoni, Anna Malpaga, Marco Miotto, Vicki L. J. Whitehall, Barbara A. Leggett, Janelle L. Harris, Jonathan Harris, Marc D. Jones, Jeremy Humphris, Lorraine A. Chantrill, Venessa Chin, Adnan M. Nagrial, Marina Pajic, Christopher J. Scarlett, Andreia Pinho, Ilse Rooman, Christopher Toon, Jianmin Wu, Mark Pinese, Mark Cowley, Andrew Barbour, Amanda Mawson, Emily S. Humphrey, Emily K. Colvin, Angela Chou, Jessica A. Lovell, Nigel B. Jamieson, Fraser Duthie, Marie-Claude Gingras, William E. Fisher, Rebecca A. Dagg, Loretta M. S. Lau, Michael Lee, Hilda A. Pickett, Roger R. Reddel, Jaswinder S. Samra, James G. Kench, Neil D. Merrett, Krishna Epari, Nam Q. Nguyen, Nikolajs Zeps, Massimo Falconi, Michele Simbolo, Giovanni Butturini, George Van Buren, Stefano Partelli \& Matteo Fassan, Australian Pancreatic Cancer Genome Initiative, Kum Kum Khanna, Anthony J. Gill, David A. Wheeler, Richard A. Gibbs, Elizabeth A. Musgrove, Claudio Bassi, Giampaolo Tortora, Paolo Pederzoli, John V. Pearson, Nicola Waddell, Andrew V. Biankin \& Sean M. Grimmond

Nature 543, 65-71 (2017); doi:10.1038/nature21063

It has been brought to our attention that in Fig. $2 \mathrm{~d}$ of this Article, an incorrect Sanger trace was used to represent the breakpoint of the EWSR 1 and FLI1 type 2 fusion. This was due to an error during manuscript preparation, when we inadvertently inserted the electrophoretic trace referring to EWSR1 splicing variants. Figure 2d has been corrected in the online versions of the Article. We apologize for the confusion.

In addition, in the first sentence of the 'Somatic driver mutation' section on page 67, the sentence: 'A total of 15,751 somatic coding mutations (7,703 non-silent) were detected in 2,787 genes (Supplementary Tables 4, 5)", should have stated "A total of 3,097 somatic coding mutations (2,498 non-silent) were detected in 2,567 genes (Supplementary Tables 4,5 )". Note that the numbers in the Supplementary Tables are correct. This sentence has been corrected in the online versions of the Article. 